\title{
ABORDAGENS TEÓRICAS SOBRE A SAÚDE DO TRABALHADOR DE ENFERMAGEM: REVISÃO INTEGRATIVA*
}

Elaine Cristina Novatzki Forte', Ana Paula Trombetta', Denise Elvira Pires de Pires², Francine de Lima Gelbcke ${ }^{3}$, Murielk Motta Lino ${ }^{4}$

${ }^{1}$ Enfermeira. Doutoranda em Enfermagem. Universidade Federal de Santa Catarina. Florianópolis-SC-Brasil. ${ }^{2}$ Enfermeira. Doutora em Ciências Sociais. Universidade Federal de Santa Catarina. Florianópolis-SC-Brasil. ${ }^{3}$ Enfermeira. Doutora em Enfermagem. Universidade Federal de Santa Catarina. Florianópolis-SC-Brasil. ${ }^{4}$ Enfermeira. Mestre em Enfermagem. Eletrosul Centrais Elétricas S.A. Florianópolis-SC-Brasil.

RESUMO: Revisão integrativa com o objetivo de identificar as abordagens teóricas referidas por autores de estudos atuais sobre a saúde do trabalhador de enfermagem. A busca das publicações entre 2008 e 2012 ocorreu em três bases de dados, utilizando-se os descritores saúde do trabalhador e enfermagem. A amostra constituiu-se de 18 artigos. Dentre os artigos excluídos, por não conterem a abordagem teórica explicitada, encontraramse estudos quantitativos, estudos transversais e de coorte. Os estudos selecionados possuem características semelhantes, relacionadas à subjetividade do trabalhador e as formas de organização do trabalho. As abordagens teóricas enriquecem a discussão sobre prazer e sofrimento no trabalho de enfermagem, ofertando um leque de possibilidades de interpretação para as situações vivenciadas pelos trabalhadores, e que independentemente da teoria adotada, os estudos corroboram entre si, no sentido de enriquecer o debate acerca da temática, alguns levantando situações emergentes, outros fornecendo subsídios para a melhoria das condições de trabalho.

DESCRITORES: Saúde do trabalhador; Força de trabalho; Recursos humanos de enfermagem.

\section{THEORETICAL APPROACHES IN OCCUPATIONAL HEALTH OF NURSING WORKERS: AN INTEGRATIVE REVIEW}

\begin{abstract}
This integrative review aims to identify the theoretical approaches mentioned by authors in studies about the health of nursing workers. The search for publications between 2008 and 2012 occurred in three databases, using the descriptors of 'occupational health' and 'nursing'. The sample was made up of 18 articles which dealt specifically with the occupational health of nursing workers. Of the studies selected, the vast majority did not make clear the theoretical framework adopted, and among those which did, two approaches predominated: work and subjectivity, and health policies. The different theoretical approaches enriched the discussion showing the strong relation between organization, work conditions, and subjectivity and situations of pleasure and suffering in the nursing work.
\end{abstract}

DESCRIPTORS: Occupational health; Workforce; Nursing human resources.

\section{ABORDAJES TEÓRICOS ACERCA DE LA SALUD DEL TRABAJADOR DE ENFERMERÍA: REVISIÓN INTEGRATIVA}

RESUMEN: Revisión integrativa con el objetivo de identificar los abordajes teóricos referidos por autores de estudios actuales sobre la salud del trabajador de enfermería. La búsqueda de las publicaciones entre 2008 y 2012 ocurrió en tres bases de datos, utilizándose los descriptores salud del trabajador y enfermería. La muestra fue constituida de 18 artículos. Entre los artículos excluidos, por no contener el abordaje teórico explicitado, están estudios cuantitativos, estudios transversales y de cohorte. Los estudios seleccionados poseen características semejantes, relacionadas a la subjetividad del trabajador y las formas de organización del trabajo. Los abordajes teóricos enriquecen la discusión sobre placer y sufrimiento en el trabajo de enfermería, ofreciendo una serie de posibilidades de interpretación para las situaciones vivenciadas por los trabajadores, y que independientemente de la teoría adoptada, los estudios corroboran entre sí, en el sentido de enriquecer el debate acerca de la temática, algunos verificando situaciones, otros proveyendo subsídios para la mejoría de las condiciones de trabajo.

DESCRIPTORES: Salud del trabajador; Fuerza de trabajo; Recursos humanos de enfermería.

*Artigo originado na disciplina Processo de Trabalho em Saúde e Enfermagem do Programa de Pós-

Graduação em Enfermagem. Universidade Federal de Santa Catarina, 2013.

Autor Correspondente:

Elaine Cristina Novatzki Forte

Universidade Federal de Santa Catarina

Rua Jurerê Tradicional, 1097 - 88053-750 - Florianópolis-SC-Brasil

E-mail: elainecnforte@gmail.com
Recebido: $16 / 02 / 2013$

Finalizado: 18/07/2014 


\section{INTRODUÇÃO}

O trabalho humano acontece em função de uma transformação planejada de algo, com o objetivo de atender às necessidades humanas ${ }^{(1)}$. A 'saúde do trabalhador' constitui uma área da saúde pública que tem como objeto de estudo e intervenção as relações entre o trabalho e a saúde. Tem como objetivos a promoção e a proteção da saúde do trabalhador. As intervenções ocorrem através de ações de vigilância dos riscos presentes nos ambientes e condições de trabalho, dos agravos à saúde do trabalhador, organização e prestação da assistência aos trabalhadores.

Os trabalhadores da enfermagem, inseridos em diferentes organizações institucionalizadas, convivem com diferentes cargas de trabalho e estão expostos a diferentes situações de risco ocupacional. Sabe-se que o trabalho de enfermagem é desgastante, uma vez que, além de lidar com a dor, o sofrimento e a morte, mantém contato com uma variedade de riscos físicos, químicos, biológicos, ergonômicos e de acidentes, os quais lhe impõem situações que podem levar ao adoecimento( ${ }^{(2)}$. Deste modo, as relações de trabalho e condições de trabalho podem interferir na saúde dos profissionais de enfermagem, pois influenciam diretamente no aparecimento e/ou desenvolvimento de doenças em função de situações de risco como estresse e sobrecarga de trabalho, aos quais muitos profissionais são expostos ${ }^{(3)}$.

As instituições de saúde são tipicamente insalubres, e ainda há limitações para instituir novas formas de organização do trabalho da enfermagem. No entanto, torna-se possível controlar a insalubridade, a periculosidade e a penosidade, assim como o desgaste e a exaustão dos trabalhadores, permitindo a recuperação da força de trabalho(4). Nesse sentido, estudos diversos são propostos com vistas a compreender este contexto em enfermagem, na expectativa de contribuir para uma força de trabalho mais saudável. Para tanto, as teorias que se propõe a analisar o trabalho humano auxiliam nesta compreensão.

Com base no exposto, surgiu a motivação de realizar uma Revisão Integrativa para identificar em estudos atuais, acerca da saúde do trabalhador de enfermagem, as abordagens teóricas utilizadas, a fim de identificar os olhares utilizados para a saúde do trabalhador de enfermagem. Ao identificar as abordagens teóricas utilizadas, estudiosos dessa temática poderão ter novas concepções para desenvolver novos estudos. Para tanto, o objetivo deste estudo é identificar quais abordagens teóricas são referidas pelos autores de estudos atuais sobre a saúde do trabalhador de enfermagem.

\section{MÉTODO}

Estudo exploratório-descritivo, que segue os pressupostos da revisão integrativa ${ }^{(5)}$. As bases de dados pesquisadas foram determinadas por assunto (de saúde e de enfermagem), sendo elas: Cumulative Index to Nursing and Allied Health Literature (CINAHL), Literatura Latino-Americana em Ciências da Saúde (LILACS) e Scientific Electronic Library Online (SciELO) e os estudos escolhidos na forma artigos de periódicos, com recorte temporal de cinco anos (2008-2012), com a intenção de levantar dados atuais disponíveis on-line e na íntegra.

A pesquisa ocorreu entre 12 de dezembro de 2012 a 15 de fevereiro de 2013 e os idiomas escolhidos foram Português, Espanhol e Inglês. Foi elaborado um cruzamento de palavras-chave (saúde do trabalhador e enfermagem), com o operador booleano AND nas caixas de busca que se referem a assunto e palavras do resumo, respectivamente. Um total de 594 referências foi recuperado a partir das bases de dados.

Dois critérios de elegibilidade foram aplicados aos resumos dos estudos: a) estudos especificamente relacionados à saúde dos profissionais de enfermagem; b) foram excluídos alguns tipos de estudos/publicações: tese, dissertação, carta, resenha, artigos de revisão, livros, capítulos de livros, documentos governamentais, boletins informativos.

Das 594 referências, 23 (2,9\%) foram excluídas por não disponibilizar o texto completo, 212 $(35,6 \%)$ por não atenderem aos critérios de inclusão previamente estabelecidos no protocolo de revisão de literatura e 225 (57,07\%) por não cumprir um dos dois critérios de elegibilidade. Um total de 134 referências foi aceito para a fase seguinte. Destas 09 foram duplicadas e, portanto, excluídas. O número final de estudos foi de 125. 
Os trabalhos pré-selecionados foram organizados em uma matriz com os seguintes itens: número de ordem, título do estudo, país de estudo, base de dados e abordagem teórica utilizada. A análise dos estudos seguiu os passos para análise de dados qualitativos, com préanálise, exploração do material e tratamento e interpretação dos resultados ${ }^{(6)}$. Os estudos foram agregados a fim de estruturar as abordagens teóricas adotadas nos estudos atuais sobre a saúde do trabalhador de enfermagem.

Por se tratar de uma revisão integrativa e não haver envolvimento direto de seres humanos, as questões éticas a serem analisadas por um Comitê de Ética em Pesquisas com Seres Humanos foram dispensadas.

\section{RESULTADOS}

Um total de 125 estudos foi incluído, dos quais 48 (39\%) foram encontrados na base de dados CINAHL, 30 (24\%) na base de dados da SCIELO e $47(37 \%)$ na base de dados da LILACS. Desse total, 108 estudos não tiveram a abordagem teórica explicitada por seus autores, o que não representa que esses estudos não o contenham, entretanto, não foi incluído na análise final.

Dentre os artigos excluídos, por não conterem a abordagem teórica explicitada, encontraram-se estudos quantitativos, estudos transversais e de coorte, com objetivos distintos. Alguns utilizaram escalas de satisfação e qualidade de vida, outros tratavam de estudos epidemiológicos, nos quais se observou a incidência de algumas patologias relacionadas ao trabalho da enfermagem. Em outros estudos, a abordagem teórica foi identificada implicitamente, entretanto, não foram incluídos na amostra final, tendo em vista o objetivo desta revisão.

Os estudos que tiveram o referencial teórico identificado somaram 18, sendo 12 do Brasil, 4 dos Estados Unidos, Inglaterra e Taiwan, com 1 estudo cada Quadro 2.

O fato de o maior número de publicações serem do Brasil, se deve a escolha das bases de dados, a base de dados LILACS contém referências bibliográficas na área da Saúde publicadas nos países da América Latina e do Caribe e a SciELO é uma biblioteca eletrônica que abrange uma coleção selecionada de periódicos científicos brasileiros.

Os principais resultados encontrados foram compilados por referencial teórico, utilizando a análise de dados qualitativos para identificar as contribuições de cada tipo de referencial, para o desenvolvimento da temática Saúde do Trabalhador de Enfermagem.

Quadro 1 - Distribuição do número de estudos encontrados com a respectiva porcentagem de cada referencial teórico identificado. Florianópolis-SC-Brasil, 2013

\begin{tabular}{|l|c|}
\hline Referencial Teórico Adotado & $\mathbf{n} / \%$ \\
\hline Referencial não identificado & $108 / 86,4 \%$ \\
\hline Materialismo Histórico Dialético/Cargas de Trabalho & $03 / 2,4 \%$ \\
\hline Farmacologia e Promoção da Saúde & $01 / 0,8 \%$ \\
\hline Fenomenologia & $01 / 0,8 \%$ \\
\hline Trabalho e Subjetividade & $05 / 4 \%$ \\
\hline Políticas de Saúde & $06 / 4,8 \%$ \\
\hline Modelo de crenças em saúde de Rosenstock & $01 / 0,8 \%$ \\
\hline Necessidades Humanas Básicas de Maslow & $01 / 0,8 \%$ \\
\hline
\end{tabular}


Quadro 2 - Distribuição dos artigos segundo autores, país, base de dados e abordagem teórica. FlorianópolisSC-Brasil, 2013

\begin{tabular}{|c|c|c|c|}
\hline Autores & País & Base de Dados & Abordagem Teórica Utilizada \\
\hline $\begin{array}{l}\text { MARTINS, J.T.; ROBAZZI, M.L.C.C.; BOBROFF, } \\
\text { M.C.C. }\end{array}$ & Brasil & SciELO & \multirow{5}{*}{$\begin{array}{l}\text { Trabalho e Subjetividade/ } \\
\text { Foucault }\end{array}$} \\
\hline GARCIA, A.B. et al & Brasil & SciELO & \\
\hline CAMPOS, J.F.; DAVID, H.S.L. & Brasil & LILACS & \\
\hline SOARES, R.J.O. et al & Brasil & SciELO & \\
\hline GUAZINA, L.; TITTONI, J. & Brasil & LILACS & \\
\hline AZAMBUJA, E.P. et al & Brasil & SciELO & \multirow{3}{*}{$\begin{array}{l}\text { Materialismo Histórico- } \\
\text { Dialético/Cargas de Trabalho }\end{array}$} \\
\hline SECCO, I. A.O. et al & Brasil & LILACS & \\
\hline MININEL, V.A.; BAPTISTA, P.C.P.; FELLI, V.E.A. & Brasil & CINAHL & \\
\hline REGIS, L.F.L.V.; PORTO, I.S. & Brasil & SciELO & $\begin{array}{l}\text { Necessidades Humanas } \\
\text { Básicas de Maslow } \\
\end{array}$ \\
\hline NEVES, H.C.N. et al. & Brasil & LILACS & $\begin{array}{l}\text { Modelo de Crenças em Saúde } \\
\text { de Rosenstock }\end{array}$ \\
\hline DIAS, J.R.F. et al. & Brasil & LILACS & $\begin{array}{l}\text { Farmacologia e Promoção da } \\
\text { Saúde }\end{array}$ \\
\hline BOBROFF, M.C.C.; MARTINS, J.T. & Brasil & LILACS & \multirow{6}{*}{ Políticas de Saúde } \\
\hline AZIZ, A. M. & Inglaterra & CINAHL & \\
\hline FARIS, J. & EUA & CINAHL & \\
\hline MENZEL, N. N. & EUA & CINAHL & \\
\hline WEISS, M. D.; BERNARDS, P.; PRICE, S.J. & EUA & CINAHL & \\
\hline LIN, Y. P.; HONG, O.; YEH, M. C. & Taiwan & CINAHL & \\
\hline LETVAK, S. & EUA & CINAHL & Fenomenologia \\
\hline
\end{tabular}

\section{DISCUSSÃO}

\section{Trabalho e Subjetividade}

A subjetividade no trabalho da enfermagem foi tratada de forma distinta nos estudos. A musicoterapia foi associada à redução do estresse, fazendo com que o trabalhador se refaça, retomando melhores condições físicas e psíquicas para retornar ao trabalho. Em outro estudo, que buscou identificar os fatores facilitadores e impeditivos no prazer no trabalho de técnicos em enfermagem, os fatores impeditivos se sobrepõe aos facilitadores, gerando um obstáculo para o cuidado de si e consequente desmotivação e prejuízos para a saúde dos trabalhadores.

A satisfação no trabalho é expressa em 3 dos 5 estudos que abordaram a temática da subjetividade. Esta é entendida como prazer no trabalho e os principais motivos de satisfação no trabalho da enfermagem são: a remuneração, o gostar do que faz, reconhecimento, trabalho em equipe, o "poder salvar vidas"; em contrapartida, os principais motivos de insatisfação no trabalho são: baixa ou nenhuma autonomia, gestão não participativa, falta de apoio da supervisão, falta de comunicação da equipe, sobrecarga de trabalho, condições de trabalho, violência e sofrimento dos pacientes. Alguns estudos destacaram que a satisfação no trabalho da enfermagem é preditora de maior retenção de trabalhadores no serviço, menor índice de absenteísmo e menor rotatividade.

Essa abordagem teórica permitiu uma melhor compreensão da subjetividade impressa no trabalho da enfermagem, mas há uma limitação em avaliar com profundidade as influências do trabalho na saúde do trabalhador, devido à subjetividade e complexidade envolvidas, como saúde, sofrimento, doença, prazer, entre outros.

A partir dos estudos encontrados, pode-se observar que, muitas das queixas subjetivas dos profissionais, principalmente as relacionadas com situações conflitantes, seriam melhor trabalhadas, ou até mesmo resolvidas se o profissional de enfermagem recebesse um suporte emocional especializado. 


\section{Materialismo Histórico-Dialético e Cargas de Trabalho}

O trabalho é categoria central de análise do Materialismo Histórico, pois é a forma mais simples e objetiva para se organizar em sociedade, e a base das relações sociais são as de produção, as formas de organização do trabalho.

A importância de analisar o contexto históricosocial, no qual o trabalho de enfermagem é realizado, se deve à existência de macro e microdeterminantes que influenciam na forma como o trabalho se desenvolve, bem como na sua divisão, podendo gerar prazer/satisfação, sofrimento e/ou adoecimento ${ }^{(25)}$.

Diferente de outros setores da produção capitalista, a produção em saúde é operada de forma específica, e a enfermagem como trabalho teve fortes influências das alterações no mundo do trabalho no setor saúde.

Os principais achados nos estudos se referem ao processo de trabalho das equipes de enfermagem e às cargas físicas e psíquicas dos trabalhadores de enfermagem. O desgaste físico aparece com maior frequência nos auxiliares e técnicos em enfermagem, nos quais os distúrbios osteomusculares são uma constante. A dor é o fator de maior comprometimento do trabalho desses profissionais (coluna, pernas, pés e cabeça), sendo também, o maior motivo de absenteísmo. $\mathrm{O}$ estresse e o desgaste psíquico são mais frequentes entre os enfermeiros e pode ser ocasionado por inúmeros fatores, dentre eles: forma de organização no trabalho, jornadas de trabalho de 40 horas ou mais, trabalho noturno e convivência com situações estressantes (morte, sofrimento e outros). As cargas de trabalho psíquicas de enfermagem se manifestam por meio dos contingentes insuficientes de pessoal, do trabalho pouco reconhecido e mal remunerado, da complexidade técnica, tecnológica e das interrelações pessoais inerentes a essa atividade, o que contribui para as doenças emocionais.

\section{Necessidades Humanas Básicas de Maslow}

A teoria de Maslow é conhecida como uma das mais importantes teorias de motivação. Segundo ele, as necessidades dos seres humanos obedecem a uma hierarquia, isso significa que a partir do momento em que uma pessoa atende uma necessidade, surge outra em seu lugar, concretizando um ciclo(15). Quando, por algum motivo, a necessidade não é satisfeita, de alguma maneira ela será transferida ou compensada, portanto, a motivação é um estado cíclico e constante na vida pessoal.

Oestudo encontrado indica que as necessidades humanas básicas da equipe de enfermagem estão comprometidas, principalmente as necessidades de segurança e fisiológicas, as mais primárias. Com isso, a equipe de enfermagem tende a reproduzir suas insatisfações na relação do cuidado com o outro, comprometendo-o, e que, portanto, as instituições de saúde devem saber reconhecer os fatores estressantes no trabalho, a fim de procurar e incentivar estratégias de enfrentamento com vistas a minimizar o estresse profissional.

\section{Políticas de Saúde}

Os achados mais frequentes nos estudos que referem as Políticas de Saúde tratam da educação continuada (treinamentos, capacitação dos profissionais, adesão aos EPIs e Programas de Educação Permanente e Minimização de Riscos Ocupacionais). Mudanças nas políticas para minimizar os estressores ocupacionais também foram citadas. Algumas formas para evitar o adoecimento no trabalho foram vastamente citadas (uso de EPI, seguimento clínico pósexposição a material biológico ou acidente com perfuro-cortante, formas de manejo do estresse, redistribuição do trabalho por turnos) como estratégias a serem adotadas para melhorar as políticas existentes.

\section{Modelo de Crenças em Saúde de Rosenstock}

O modelo proposto por Rosenstock ${ }^{(16)}$ considera a relação entre a percepção que uma pessoa tem de sua suscetibilidade para contrair uma doença e da severidade da doença para si. É utilizada para compreender melhor os fatores que influenciam na percepção e nas crenças das pessoas, a fim de obter ou manter os cuidados com a saúde.

Os trabalhadores de enfermagem conhecem os riscos a que estão expostos no ambiente de 
trabalho. O estudo em questão revelou que os trabalhadores compreendem que o não uso dos EPIs aumenta as chances de adquirir doenças ocupacionais e que estes têm consciência de que seu uso não exclui o risco de infecção por patógenos. O estudo revelou, também, que a baixa adesão ao uso dos EPIs está associada aos aspectos organizacionais, gerenciais e relacionais, como: estrutura física inadequada, disponibilidade e acessibilidade de EPIs, falta de rotinas, sobrecarga de trabalho, estresse, improvisação e desgaste nas relações de trabalho.

\section{Farmacologia e Promoção da Saúde}

A equipe de enfermagem, em específico, a hospitalar, são mais vulneráveis aos agravos à saúde, tanto na dimensão física quanto na psíquica e isso se deve a um rol de fatores, como: as condições precárias de trabalho, jornadas de trabalho exaustivas e as dificuldades cotidianas, o que favorece o uso de substâncias psicoativas(26).

O contato constante com os pacientes e a exposição a várias situações compromete a saúde física, emocional e mental. Essa vulnerabilidade explica o alto índice de estresse, depressão, cansaço e outras doenças ocupacionais. Por falta de mecanismos e estratégias de enfrentamento de tais situações, os trabalhadores recorrem, muitas vezes, à automedicação, que no Brasil é uma prática bastante comum ${ }^{(27)}$.

A utilização de psicotrópicos pelos trabalhadores de enfermagem é comum. No estudo encontrado referente a essa temática, ressalta-se que, os fatores que favorecem o consumo são: período de alto estresse, cobranças múltiplas e insatisfação com o setor de trabalho ou equipe e a família, o que torna necessário que os profissionais tenham melhor qualidade de vida e trabalho para poder evitar essa problemática.

\section{Fenomenologia}

A fenomenologia é uma ciência subjetiva, que estuda o próprio fenômeno, visando reconhecer e esclarecer o fenômeno, a partir de estudos que consideram a visão de um determinado sujeito espectador $^{(28)}$.

Busca-se, na fenomenologia, a essência de um determinado fenômeno através do processo de redução fenomenológica e que todas as coisas são consideradas inacabadas e estão em constante processo de modificação. A fenomenologia é um tratado científico a respeito da descrição e classificação dos fenômenos.

Os estudos recentes também mostram a preocupação com a segurança do paciente, especialmente, quanto ao tempo de resposta na assistência, pois a evolução do paciente influencia a saúde dos enfermeiros, no sentido de que se o paciente evolui mal, o enfermeiro tende a se sobrecarregar, física e psicologicamente. Destarte, as instituições de saúde devem disponibilizar apoio no ambiente de trabalho, especialmente o apoio psicológico para enfrentar a dor e a depressão.

O estudo encontrado que utilizou a abordagem fenomenológica destaca que uma força de trabalho de enfermagem envelhecida impacta diretamente na qualidade da assistência prestada ao paciente, e que pesquisas têm mostrado que a manutenção dessas enfermeiras mais velhas exigem algumas medidas de manutenção da saúde, como: mais controle sobre o ambiente de trabalho, reconhecimento no trabalho, horários de trabalho favoráveis, incentivos econômicos, empregos menos extenuantes que utilizam a experiência e educação, medidas ergonômicas no ambiente de trabalho e programas de aposentadoria melhores.

\section{CONSIDERAÇÕES FINAIS}

$\mathrm{Na}$ busca para entender melhor quais as abordagens teóricas foram utilizadas por autores atuais nos estudos referentes à saúde do trabalhador de enfermagem, algumas teorias emergiram desse assunto específico, com características únicas relacionadas à subjetividade do trabalhador e todas as formas de organização em que o trabalho acontece.

O sucesso e o bom desempenho das instituições de saúde dependem, dentre outros fatores, de trabalhadores saudáveis e motivados para o trabalho. Trabalhadores estressados e/ou com doenças ocupacionais têm a qualidade da assistência e a produtividade comprometidas, assim como, as instituições, pois deixam de prestar serviços de qualidade, devido aos altos 
índices de absenteísmo e licenças de saúde.

As abordagens teóricas encontradas nos estudos desta revisão enriquecem a discussão sobre prazer e sofrimento no trabalho de enfermagem, ofertando um leque de possibilidades de interpretação para as situações vivenciadas pelos trabalhadores.

Importante ressaltar que, mesmo adotando teorias diferentes, os estudos corroboram entre si, no sentido de enriquecer o debate acerca da temática, alguns levantando situações emergentes, outros fornecendo subsídios para a melhoria das condições de trabalho.

Observa-se ainda, que apesar do expressivo número de publicações encontradas sobre a saúde do trabalhador, estudos ainda se fazem necessários, tendo em vista a complexidade desta temática, e que sob novos olhares podem contribuir para uma enfermagem mais saudável, sem tantos sofrimentos.

\section{REFERÊNCIAS}

1. Pires DEP. A enfermagem enquanto disciplina, profissão e trabalho. Rev. bras. enferm. 2009; 62(5):739-44.

2. Carvalho LSF, Matos RCS, Souza NVDO, Ferreira REDS. Motivos de afastamento por licença de saúde dos trabalhadores de enfermagem. Cienc. cuid. saúde. 2010;9(1):60-6.

3. Duarte MLC, Avelhaneda JC, Parcianello RR. A saúde do trabalhador na estratégia de saúde da família: percepções da equipe de enfermagem. Cogitare enferm. 2013;18(2):323-30.

4. Felli VEA. Condições de trabalho de enfermagem e adoecimento: motivos para a redução da jornada de trabalho para 30 horas. Enferm. foco. 2012;3(4):178-81.

5. Whittemore R, Knafl $\mathrm{K}$. The integrative review: updated methodology. J. adv. nurs. 2005;52(5):546-53.

6. Minayo MCS. O desafio do conhecimento: pesquisa qualitativa em saúde. 12a ed. São Paulo: HucitecAbrasco; 2010.

7. Martins JT, Robazzi MLCC, Bobroff MCC. Prazer e sofrimento no trabalho da equipe de enfermagem: reflexão à luz da psicodinâmica Dejouriana. Rev Esc Enferm USP. 2010;44(4):1107-11.

8. Garcia AB, Dellaroza MSG, Haddad MCL, Pachemshy LR. Prazer no trabalho de técnicos de enfermagem do pronto-socorro de um hospital universitário público. Rev. Gaúcha Enferm. 2012;33(2):153-59.
9. Campos JF, David HSL. Avaliação do contexto de trabalho em terapia intensiva sob o olhar da psicodinâmica do trabalho. Rev Esc Enferm USP. 2011;45(2):36-8.

10. Soares RJO, Zeitoune RCG, Lisboa MTL, Mauro MYC. Fatores facilitadores e impeditivos no cuidar de si para docentes de enfermagem. Texto Contexto Enferm. 2011;20(4):758-65.

11. Guazina L, Tittoni J. Musicoterapia institucional na saúde do trabalhador: conexões, interfaces e produções. Psicol. Soc. 2009;21(1):108-17.

12. Azambuja EP, Pires DEP, Vaz MRC, Marziale MH. É possível produzir saúde no trabalho da enfermagem? Texto Contexto Enferm. 2010;19(4):658-66.

13. Secco IAO, Robazzi MLCC, Souza FEA, Shimizu DS. Cargas psíquicas de trabalho e desgaste dos trabalhadores de enfermagem de hospital de ensino do Paraná. SMAD, Rev. Eletr. Saúde Mental Alcool Drog. 2010;6(1):1-17.

14. Mininel VA, Baptista PCP, Felli VEA. Cargas psíquicas e processos de desgaste em trabalhadores de enfermagem de hospitais universitários brasileiros. Rev. Latino-Am. Enferm. [Internet] 2011;19(2) [acesso em 22 fev 2013]. Disponível: http://dx.doi.org/10.1590/ S0104-11692011000200016

15. Regis LFLV, Porto IS. Necessidades humanas básicas dos profissionais de enfermagem: situações de (in)satisfação no trabalho. Rev Esc Enferm USP. 2011;45(2):334-41.

16. Neves HCC, Souza ACS, Medeiros M, Munari DB, Ribeiro LCM, Tipple AFV. Segurança dos trabalhadores de enfermagem e fatores determinantes para adesão aos equipamentos de proteção individual. Rev. Latino-Am. Enferm. [Internet] 2011;19(2) [acesso em 22 fev 2013]. Disponível: http://dx.doi.org/10.1590/ S010411692011000200018

17. Dias JRF, Araújo CS, Martins ERC, Clos AC, Francisco MTR, Sampaio CEP. Fatores predisponentes ao uso próprio de psicotrópicos por profissionais de enfermagem. Rev. enferm. UERJ. 2011;19(3):445-51.

18. Bobroff MCC, Martins JT. Aspectos éticoslegais da equipe de enfermagem em saúde do trabalhador: reflexões necessárias. Cienc. cuid. saude. 2011;10(3):608-13.

19. Aziz AM. Subcutaneous injections: preventing needlestick injuries in the community. Br. J. Community Nurs. 2012;17(6):258-64.

20. Faris J. Lowering nursing injuries using post offer preemployment testing. Work 2008;31(1):39-45.

21. Menzel NN. Underreporting of musculoskeletal 
disorders among health care workers. AAOHN J. 2008;56(12):487-94.

22. Weiss MD, Bernards P, Price SJ. Working through a migraine addressing the hidden costs of workplace headaches. J. Contin. Educ. Nurs. 2008;56(12):495500.

23. Lin YP, Hong O, Yeh MC. Occupational health nursing practice, education, and research in Taiwan. AAOHN J. 2008;56(4):151-8.

24. Letvak S. Hurting at work: the lived experience of older nurses. Int. j. human caring. 2009;13(4):8-16.

25. Gonçalves ASR. Prazer e sofrimento no trabalho de docentes da saúde em Universidade Pública da Região Amazônica [tese]. Florianópolis (SC): Universidade Federal de Santa Catarina; 2010.

26. Moutinho ECVS, Lopes GT. Enfermeiro do programa saúde da família: conceitos e crenças sobre drogas e modelos teóricos explicativos. Rev. enferm. UERJ. 2008;16:51-7.

27. Baggio MA, Formaggio FM. Automedicação: desvelando o descuidado de si dos profissionais de enfermagem. Rev. enferm. UERJ. 2009;17:224-8.

28. Finlay L. Debating phenomenological research methods. Phenomenol. Practice. 2009;3(1):6-25. 